\title{
ASPIRAÇÃO ENDOTRAQUEAL EM PACIENTES COM VIA AÉREA ARTIFICIAL SOB VENTILAÇÃO MECÂNICA INVASIVA INTERNADOS EM UTI
}

\section{ENDOTRACHEAL SUCTION IN PATIENTS HOSPITALIZED IN ICU WITH ARTIFICIAL AIRWAY IN INVASIVE MECHANIC VENTILATION}

\section{ASPIRACIÓN ENDOTRAQUEAL EM PACIENTES CON VÍA AÉREA ARTIFICIAL BAJO VENTILACIÓN MECÁNICA INVASORA INTERNADOS EN UCI}

Vagner José Lopes ${ }^{1}$, Francielle Muller ${ }^{2}$, Marli Aparecida Rocha de Souza ${ }^{3}$, Izabela Andrea da Silva ${ }^{4}$

\section{RESUMO}

Objetivo: Identificar e analisar as intervenções e o manejo da enfermagem na aspiração endotraqueal em pacientes adultos com via aérea artificial em ventilação mecânica, internados em unidade de terapia intensiva. Método: Trata-se de uma revisão integrativa de literatura, com abordagem quantitativa, realizada nas bases de dados Literatura Latino-Americana e do Caribe em Ciências da Saúde e PubMed, por meio dos seguintes descritores: "enfermagem and sucção and unidades de terapia intensiva" e "nursing and suction and intensive care units", entre os anos de 2012 e 2017. Resultados: A busca nas bases de dados resultou em 108 artigos; após a aplicação dos critérios de inclusão e exclusão e a análise de conteúdo, nove artigos foram selecionados. Identificaram-se como intervenção 16 cuidados de enfermagem referentes à prevenção de infecções, colonização bacteriana, alterações hemodinâmicas e respiratórias e monitorização do paciente durante e após o procedimento de aspiração da cânula endotraqueal. Conclusão: As intervenções encontradas são relevantes para a prática clínica de enfermagem na aspiração endotraqueal, porém há a necessidade de avaliação, por meio do exame físico do paciente antes do procedimento, a fim de facilitar a escolha da técnica ideal e assegurar a tomada de decisão do enfermeiro para a execução do procedimento.

Descritores: Enfermagem; Sucção; Unidades de terapia intensiva.

\section{ABSTRACT}

Objective: To identify and to analyze nursing interventions and management in endotracheal suction in adult patients with artificial airway in mechanical ventilation, hospitalized in an intensive care unit. Method: It is an integrative literature review , with quantitative approach, carried out in the Latin American and Caribbean Literature databases in Health Sciences and PubMed, through the following descriptors: "enfermagem and sucção and unidades de terapia intensiva", and "nursing and suction and intensive care units", between 2012 and 2017. Results: The research in the databases resulted in 108 articles; after applying the inclusion and exclusion criteria and the content analysis, nine articles were selected. They identified as intervention 16 nursing cares related to infection prevention, bacterial colonization, hemodynamic, breathing disorders and the monitoring patient during and after endotracheal cannula aspiration procedure. Conclusion: The interventions found are relevant for the nursing clinical practice in endotracheal suction; however, it should be made a patient evaluation through physical exam, before the procedure, in order to facilitate the choice of the ideal technique and to assure the nurse's decisions.

Descriptors: Nursing; Suction; Intensive care units.

\section{RESUMEN}

Objetivo: Identificar y analizar las interversiones y el manejo de los enfermeros en la aspiración endotraqueal en los pacientes adultos con vía aérea artificial en ventilación mecánica, internados en unidad de terapia intensiva. Método: Se trata de una revisión integrante de literatura, con abordaje de cuantificación, la cual se realizo con bases de datos en Literatura Latino-Americana y del Caribe en Ciencias de la Salud y PubMed, por medio de los siguientes descriptores:"enfermería and succión and unidades de terapia intensiva" y "nursing and suction and intensive care units", entre 2012 y 2017. Resultados: La búsqueda en las bases de datos resultó en 108 artículos; después de la aplicación de los criterios de inclusión y exclusión y el análisis del contenido, nueve artículos fueron elegidos. Se identificó como intervención 16 cuidados de la enfermería, los cuales se refieren a la prevención de infección, colonización bacteriana, cambios hemodinámicos y respiratorios y monitoramiento del paciente durante y después del procedimiento de aspiración de la cánula endotraqueal. Conclusión: Las intervención que fueron encuentradas son relevantes para la práctica clínica de enfermería en la aspiración endotraqueal, todavía hay la necesidad de evaluación, por medio de examen físico del paciente antes del procedimiento, con la finalidad de hacer fácil elegir la técnica ideal y dejar una seguridad mayor para el enfermero ejecutar el procedimiento.

Descriptores: Enfermería; Succión; Unidades de cuidados intensivos.

${ }^{1}$ Graduado em Enfermagem. Mestre em Tecnologia e Saúde pela Pontifícia Universidade Católica do Paraná. Docente no Centro Universitário Unidombosco, Curitiba-Pr. ${ }^{2}$ Graduada em Enfermagem pelo Centro Universitário Unidombosco, Curitiba-Pr. ${ }^{3}$ Graduada em Enfermagem. Mestre em Enfermagem pela Universidade Federal do Paraná. Docente no Centro Universitário Unidombosco, Curitiba-Pr. ${ }^{4}$ Graduada em Enfermagem. Mestre em Educação pela Universidade Tuiuti do Paraná. Docente no Centro Universitário Unidombosco, Curitiba-Pr.

Como citar este artigo:

Lopes VJ, Muller F, Souza MAR, et al. Aspiração endotraqueal em pacientes com via aérea artificial sob ventilação mecânica invasiva internados em UTI. Revista de Enfermagem do Centro-Oeste Mineiro. 2018;8:e1973. [Access Available in: http://dx.doi.org/10.19175/recom.v7i0.1973 


\section{INTRODUÇÃO}

A Aspiração Endotraqueal (AET) em pacientes adultos em Ventilação Mecânica (VM) internados em Unidade de Terapia Intensiva (UTI), é realizada por meio de técnica asséptica, utilizando um cateter flexível conectado a um sistema de vácuo e introduzido na Via Aérea Artificial (VAA) do paciente ${ }^{(1,2)}$, tendo como finalidade remover secreções pulmonares de indivíduos que são incapazes de tossir e expelir seu acúmulo, mantendo o processo de troca gasosa e melhorando a oxigenação arterial e o funcionamento pulmonar ${ }^{(1)}$.

Existem dois métodos para realizá-la: Aspiração por Sistema Fechado (ASF) e Aspiração por Sistema Aberto (ASA). Enquanto esta consiste em desconectar a VAA do paciente da VM, para a introdução da cânula flexível de aspiração, que está conectada a um sistema de vácuo, aquela é feita por meio de um equipamento chamado trachcare, que não necessita de desconexão da VAA da VM, pois funciona simultaneamente na $\mathrm{AET}^{(1)}$.

Apesar de ser um procedimento necessário para o bem-estar do paciente, pode estar sujeito a complicações, tais como: lesão na mucosa traqueal, dor, desconforto, infecção, alteração dos parâmetros hemodinâmicos e dos gases arteriais, broncoconstrição, atelectasia, aumento da pressão intracraniana, alterações do fluxo sanguíneo cerebral, ansiedade, taquicardia e hipertensão ${ }^{(3,4)}$. Sua complexidade decorre desses importantes riscos, tornando-se indispensável uma avaliação prévia do paciente. Em outras palavras, o indivíduo deve ser avaliado antes da aspiração pelos métodos de inspeção, ausculta pulmonar, percussão torácica e aferição dos sinais vitais e da Saturação de Oxigênio $\left(\mathrm{SpO}^{2}\right)$, com o intuito de verificar a necessidade de aspiração da Cânula Endotraqueal (CET) $)^{(5)}$ e sua periodicidade.

$O$ enfermeiro, nesse contexto, deve estar munido de embasamento científico sobre a teoria e prática da AET para realizá-la ${ }^{(3)}$, por ser uma atividade de rotina ao paciente crítico, com a finalidade de minimizar complicações associadas à VM. Segundo o Parecer COFEN no0557/2017, os pacientes graves, submetidos à intubação orotraqueal em UTI, deverão ter suas vias aéreas privativamente aspiradas pelo profissional Enfermeiro, conforme dispõe a Lei do Exercício Profissional da Enfermagem ${ }^{(6)}$, devido à necessidade de avaliação minuciosa do quadro clínico destes e capacidade técnica para condutas emergenciais em casos necessários ${ }^{(3)}$.

Em relação ao procedimento, estudos ${ }^{(7-9)}$ demonstram que a enfermagem desconhece algumas etapas da técnica de AET e, muitas vezes, não se baseia em evidências científicas para realizar esse cuidado, podendo causar danos e involução do paciente em seu tratamento ${ }^{(10,11)}$. Para justificar essa afirmativa, autores evidenciaram em seus estudos que os enfermeiros ainda são inseguros na teoria e prática do procedimento ${ }^{(10,12)}$, além de alguns profissionais realizarem-no sem ao menos conhecer as etapas para sua execução ${ }^{(7-9)}$, tornando-o um risco ao paciente, que deve ser avaliado adequadamente e atendido prontamente, se houver alguma intercorrência no processo de aspiração ${ }^{(10,12)}$. Ainda, resultados de estudos experimentais envolvendo enfermeiros intensivistas ${ }^{(13-15)}$ afirmam que uma avaliação do paciente pelo profissional, antes da AET, possibilita a escolha ideal da técnica e da melhor conduta a ser tomada no momento da aspiração, minimizando os riscos e complicações ao paciente em $\mathrm{VM}^{(16,17)}$.

Diante dessas evidências, formulou-se a ideia de agrupar estudos científicos que contribuíssem com o direcionamento das técnicas da prática de enfermagem na AET do paciente em VM internado em UTI, visando a reduzir complicações. Buscaram-se, assim, respostas para os principais questionamentos sobre $A E T$, por meio da seguinte questão norteadora: quais são as intervenções de enfermagem no manejo da AET do paciente adulto em estado crítico com VAA em VM, internado em UTI?

Apesar de ser um procedimento comumente realizado por estes profissionais e de sua complexidade, a AET ainda é um tema pouco abordado nos protocolos de enfermagem nos atendimentos em UTI ${ }^{(8)}$, além de não haver um consenso sobre a técnica ideal pelos profissionais enfermeiros que executam esse procedimento ${ }^{(5)}$. Esse fato está relacionado pela ausência de capacitação sobre a atualização no manejo da AET de pacientes em $\mathrm{VM}^{(8,9)}$. Desse modo, a relevância de uma revisão integrativa de literatura sobre as competências necessárias ao enfermeiro no manejo da AET justifica-se pela necessidade de evidenciar os cuidados específicos e atuais para esse procedimento, com 0 intuito de fornecer subsídios para o desenvolvimento de uma 
assistência segura ao paciente crítico na prática clínica.

Dessa forma, este estudo tem como objetivo identificar e analisar as intervenções e o manejo da enfermagem na aspiração endotraqueal em pacientes adultos com via aérea artificial em ventilação mecânica, internados em unidade de terapia intensiva.

\section{MÉTODOS}

Tratou-se de uma revisão integrativa de literatura, com abordagem quantitativa, a qual possibilitou a busca, a avaliação crítica e a sistematização de um determinado tema ${ }^{(18)}$, subsidiando, assim, o conhecimento das intervenções de enfermagem na AET em pacientes adultos críticos com VAA em VM.

A revisão foi operacionalizada em seis fases: elaboração da questão norteadora e do objetivo do estudo; estabelecimento da estratégia de busca nas bases de dados; seleção de estudos seguindo critérios de inclusão e exclusão; leitura crítica do título e resumo dos artigos selecionados; construção do instrumento de avaliação e análise dos resultados; discussão dos resultados à luz da literatura ${ }^{(19)}$.

A busca dos artigos ocorreu nas bases de dados Literatura Latino-Americana e do Caribe em Ciências da Saúde (LILACS) e PubMed, considerando artigos compreendidos entre 2012 e 2017, por meio de Descritores em Ciências da Saúde (DECSs) da Biblioteca Virtual em Saúde e dos Medical Subject Headings (MeSH), da National Library of Medicine. Utilizou-se o conectivo lógico "and" para conjugar as diferentes palavras-chave, a saber: "enfermagem and sucção and unidades de terapia intensiva" e "Nursing and suction and intensive care units". A busca foi realizada nos meses de janeiro e fevereiro de 2017.

A estratégia de seleção adotada para a busca dos artigos na segunda fase foi o método Preferred Reporting Items for Systematic Reviews and Meta-Analysis (PRISMA), considerando as quatro etapas propostas: identificação, triagem, elegibilidade e inclusão, que visam a contribuir com a qualidade dos resultados na construção do conhecimento $^{(20)}$, conforme o objetivo desta pesquisa.

Quanto ao critério de inclusão dos artigos, seu resumo e objetivos deveriam corresponder à temática proposta, sendo referenciados em português, inglês e espanhol. Por sua vez, os de exclusão foram: editoriais, dissertações, teses, artigos com referências restritas, artigos de revisão de literatura e estudos que não se encontravam no tempo cronológico definido (2012 a 2017), assim finalizando a terceira fase.

A leitura do título e resumo dos artigos, na quarta fase, foi realizada separadamente pelos pesquisadores. Qualquer discordância sobre a inclusão ou exclusão de um artigo foi resolvida pela presença de um terceiro avaliador.

A fim de realizar um processo de avaliação crítica dos estudos e apresentação dos resultados na quinta fase, desenvolveu-se um instrumento de coleta de dados elaborado pelos autores desta pesquisa e de acordo com as recomendações de estudos orientados para a elaboração de revisões integrativas ${ }^{(18,19)}$. Esse instrumento foi composto pelas seguintes variáveis: autores, ano, objetivo, metodologia, resultados e conclusão. Ainda nessa fase, após a elaboração deste instrumento, foi realizada uma análise crítica e minuciosa, a partir de uma avaliação detalhada e interpretativa dos estudos selecionados, o que permitiu a seleção dos dados relevantes para esta pesquisa e posterior análise. Ao término desta seleção, os dados foram digitados numa planilha eletrônica no programa Microsoft Excel $2010^{\circledR}$, exportados e tratados pelo pacote estatístico Statistical Package for Social Sciences ${ }^{\circledR}$ (SPSS), versão 21 , o que permitiu a análise por estatística descritiva.

Os resultados finais foram apresentados por meio de tabelas na sexta fase e discutidos à luz da literatura, de forma descritiva, com vistas a viabilizar, mediante os resultados selecionados, as intervenções evidenciadas e comparadas com outros na literatura, de maneira a responder à questão norteadora desta pesquisa.

Os aspectos éticos relacionados à revisão integrativa foram respeitados, pois os preceitos de autorias e as citações dos autores das publicações que constituíram a amostra dessa revisão foram devidamente citados.

\section{RESULTADOS E DISCUSSÃO}

Identificaram-se 108 artigos nas bases de dados PubMed e LILACS, constatando-se, após a aplicação dos critérios de inclusão/exclusão e a leitura dos títulos e resumos, que 93 não possuíam aderência à temática. Dos 15 artigos submetidos à análise de conteúdo para extração dos dados, conforme o objetivo da pesquisa, restaram nove para inclusão na revisão. A Figura 1 apresenta a estratégia de busca dos artigos nas bases de dados. 
Figura 1 - Estratégia de busca dos artigos nas bases de dados. Curitiba, Paraná, 2017.

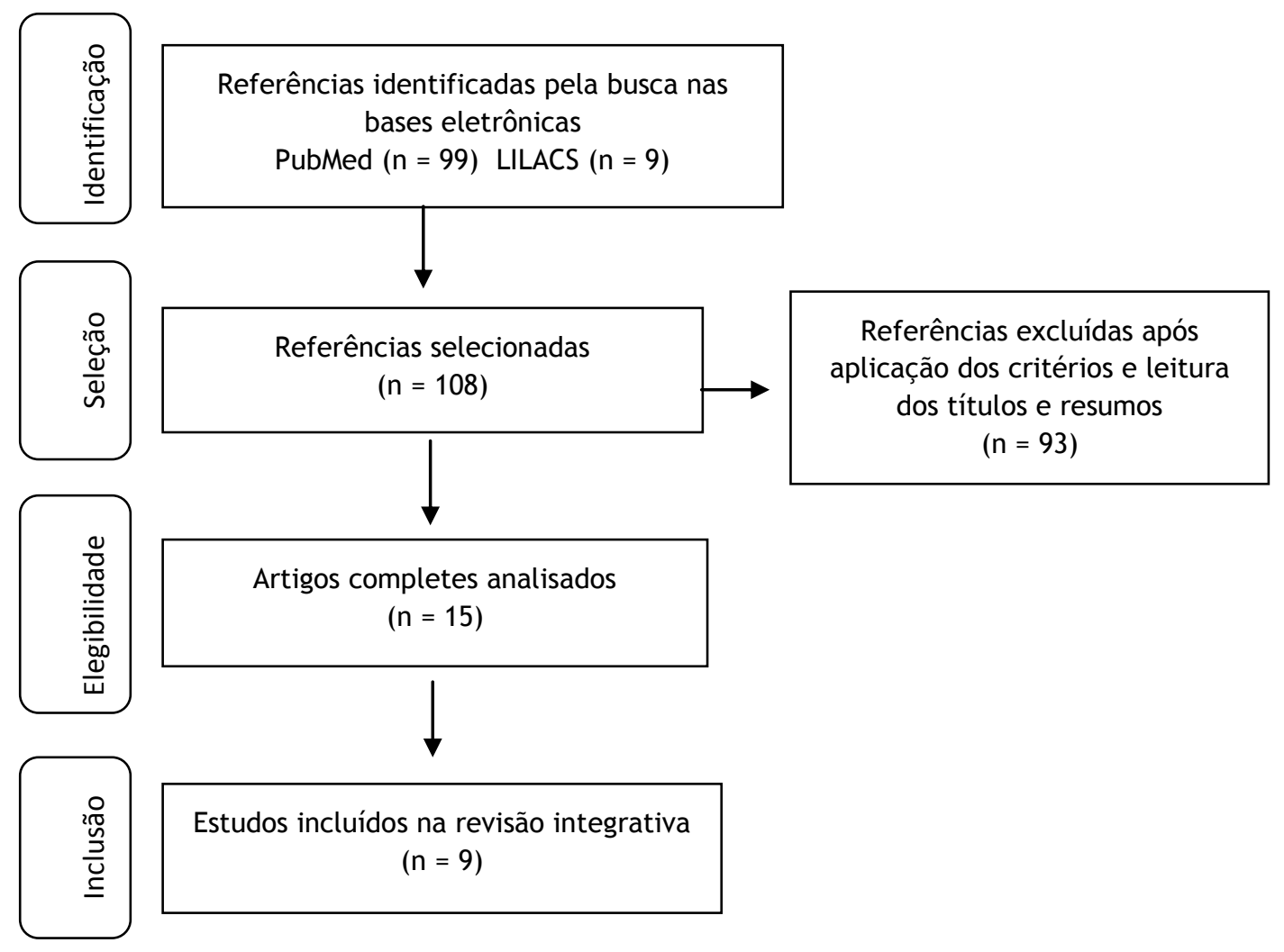

Fonte: Adaptado de Moher et al. (2009).

Em atenção ao objetivo desta pesquisa, elaborou-se uma síntese dos resultados e desfechos dos nove artigos incluídos na revisão, tendo como intenção organizar e evidenciar as intervenções de enfermagem na AET em pacientes com VAA em VM (Figura 2).

Figura 2 - Descrição dos artigos identificados nas bases pesquisadas sobre a prática de enfermagem no manejo de AET em pacientes com VAA em VM. Curitiba, Paraná, 2017.

\begin{tabular}{|c|c|c|c|c|c|c|}
\hline Artigo & Autores & Ano & Objetivo & Metodologia & Resultados & Conclusão \\
\hline$I^{(13)}$ & $\begin{array}{l}\text { Yazdannik } \\
\text { et al. }\end{array}$ & 2013 & $\begin{array}{l}\text { Comparar os efeitos } \\
\text { na troca gasosa de } \\
\text { duas diferentes } \\
\text { pressões negativas } \\
\text { por sucção, } \\
\text { aplicadas à ASF com } \\
\text { hiperoxigenação e } \\
\text { sem manobras de } \\
\text { recrutamento. }\end{array}$ & $\begin{array}{l}\text { Ensaio clínico } \\
\text { randomizado, com } \\
\text { avaliação de } \\
\text { pacientes em VM, } \\
\text { divididos em dois } \\
\text { grupos de ASF por } \\
\text { diferentes pressões } \\
\text { de sucção: } 100 \text { e } \\
200 \mathrm{mmHg} \text {. }\end{array}$ & $\begin{array}{l}\text { A análise de variância do } \\
\text { estudo não apresentou } \\
\text { diferença entre os dois } \\
\text { níveis de pressão } \\
\text { ( } p=0,315) \text {. É necessária a } \\
\text { hiperoxigenação antes, } \\
\text { durante e após o } \\
\text { procedimento. }\end{array}$ & $\begin{array}{l}\text { Pressão de sucção de } \\
200 m m H g \text { na ASF } \\
\text { não apresentou } \\
\text { efeitos prejudiciais na } \\
\text { função } \\
\text { cardiorrespiratória } \\
\text { dos pacientes. }\end{array}$ \\
\hline$\|^{(14)}$ & Yousef et al. & 2014 & $\begin{array}{l}\text { Comparar o efeito } \\
\text { de dois níveis de } \\
\text { pressão negativa na } \\
\text { ASA do tubo } \\
\text { endotraqueal de } \\
\text { pacientes } \\
\text { internados em UTI. }\end{array}$ & $\begin{array}{l}\text { Ensaio clínico } \\
\text { randomizado, } \\
\text { envolvendo pacientes } \\
\text { adultos em VM, e } \\
\text { necessitando de ASA, } \\
\text { divididos em dois } \\
\text { grupos com } \\
\text { diferentes pressões } \\
\text { de sucção: } 100 \text { e } \\
\text { 200mmHg. }\end{array}$ & $\begin{array}{l}\text { Houve diferença } \\
\text { significativa na } \mathrm{SpO}_{2} \text { e } \\
\text { frequência cardíaca após } \\
\text { a ASA dentro de cada } \\
\text { grupo }(p<0,05) \text {, porém as } \\
\text { variáveis não resultaram } \\
\text { em diferença entre os } \\
\text { dois grupos }(p>0,05) \text {. }\end{array}$ & $\begin{array}{l}\text { A sucção na ASA por } \\
\text { pressão negativa de } \\
200 \mathrm{mmHg} \text { foi } \\
\text { aplicável, com baixo } \\
\text { risco de } \\
\text { complicações. }\end{array}$ \\
\hline $\mathrm{III}^{(15)}$ & $\begin{array}{l}\text { Abbasinia et } \\
\text { al. }\end{array}$ & 2014 & $\begin{array}{l}\text { Comparar os efeitos } \\
\text { da AET por técnica } \\
\text { invasiva e }\end{array}$ & $\begin{array}{l}\text { Ensaio clínico } \\
\text { randomizado, } \\
\text { envolvendo } 74\end{array}$ & $\begin{array}{l}\text { Os pacientes } \\
\text { apresentaram alteração } \\
\text { de frequência respiratória }\end{array}$ & $\begin{array}{l}\text { A quantidade de } \\
\text { AET's no grupo de } \\
\text { aspiração invasiva }\end{array}$ \\
\hline
\end{tabular}




\begin{tabular}{|c|c|c|c|c|c|c|}
\hline & & & $\begin{array}{l}\text { minimamente } \\
\text { invasiva, em } \\
\text { pacientes } \\
\text { internados em UTI. }\end{array}$ & $\begin{array}{l}\text { pacientes, divididos } \\
\text { em dois grupos: AET } \\
\text { invasiva ( } n=37) \text { e AET } \\
\text { minimamente } \\
\text { invasiva }(n=37) .\end{array}$ & $\begin{array}{l}\text { e queda de } \mathrm{SpO}_{2} \text { logo } \\
\text { após a } \mathrm{AET} \text {, mas não } \\
\text { houve diferença } \\
\text { significativa entre os } \\
\text { grupos ( } p>0,05) \text {. Ainda, } \\
\text { verificou-se que a } \\
\text { quantidade de AET para } \\
\text { manter o tubo } \\
\text { orotraqueal permeável } \\
\text { foi maior no grupo de } \\
\text { aspiração minimamente } \\
\text { invasiva }(p<0,05) \text {. }\end{array}$ & $\begin{array}{l}\text { foi menor e, } \\
\text { consequentemente, } \\
\text { menor risco de } \\
\text { infecção por } \\
\text { exposição ao } \\
\text { procedimento. }\end{array}$ \\
\hline $\mathrm{IV}^{(16)}$ & $\begin{array}{l}\text { Irajpour et } \\
\text { al. }\end{array}$ & 2014 & $\begin{array}{l}\text { Avaliar o efeito dos } \\
\text { métodos de AET } \\
\text { invasiva e } \\
\text { minimamente } \\
\text { invasiva nos } \\
\text { parâmetros } \\
\text { cardiovasculares de } \\
\text { pacientes } \\
\text { internados em UTI. }\end{array}$ & $\begin{array}{l}\text { Ensaio clínico } \\
\text { randomizado, em } \\
\text { pacientes submetidos } \\
\text { à VM com } \\
\text { necessidade de } \\
\text { aspiração, divididos } \\
\text { em dois grupos: AET } \\
\text { invasiva e AET } \\
\text { minimamente } \\
\text { invasiva. }\end{array}$ & $\begin{array}{l}\text { Os pacientes } \\
\text { apresentaram alteração } \\
\text { de frequência cardíaca e } \\
\text { pressão arterial logo após } \\
\text { a AET em ambos os } \\
\text { grupos }(p<0,05) \text {. }\end{array}$ & $\begin{array}{l}\text { Foi evidenciada a } \\
\text { necessidade de } \\
\text { monitoramento do } \\
\text { paciente durante } \\
\text { AET, devido } \\
\text { alteração nos } \\
\text { parâmetro } \\
\text { cardiovasculares. }\end{array}$ \\
\hline $\mathrm{V}^{(9)}$ & $\begin{array}{l}\text { Hamishehka } \\
r \\
\text { et al. }\end{array}$ & 2014 & $\begin{array}{l}\text { Avaliar a adesão do } \\
\text { pacote de } \\
\text { prevenção de } \\
\text { Pneumonia } \\
\text { Associada à } \\
\text { Ventilação } \\
\text { Mecânica (PAV). }\end{array}$ & $\begin{array}{l}\text { Estudo observacional, } \\
\text { realizado em dez } \\
\text { UTI's no Irã, com } \\
\text { treinamento antes e } \\
\text { depois do pacote de } \\
\text { prevenção de PAV. }\end{array}$ & $\begin{array}{l}\text { Após o treinamento, } \\
\text { somente a técnica de } \\
\text { higiene oral com } \\
\text { gluconato de clorexedine } \\
\text { apresentou adesão pelos } \\
\text { enfermeiros. }\end{array}$ & $\begin{array}{l}\text { Recomendada a } \\
\text { ampliação da } \\
\text { educação } \\
\text { continuada e } \\
\text { supervisão dos } \\
\text { procedimentos. }\end{array}$ \\
\hline $\mathrm{VI}^{(11)}$ & $\begin{array}{l}\text { Bakhtiari et } \\
\text { al. }\end{array}$ & 2015 & $\begin{array}{l}\text { Avaliar o efeito de } \\
\text { um programa de } \\
\text { atendimento } \\
\text { respiratório de vias } \\
\text { aéreas superiores } \\
\text { na incidência de } \\
\text { PAV. }\end{array}$ & $\begin{array}{l}\text { Ensaio clínico } \\
\text { randomizado, } \\
\text { executado em } \\
\text { pacientes adultos } \\
\text { submetidos à VM e } \\
\text { internados em UTI, } \\
\text { divididos em dois } \\
\text { grupos: intervenção e } \\
\text { controle. }\end{array}$ & $\begin{array}{l}\text { As variáveis do estudo } \\
\text { (aspirações bucal e } \\
\text { subglótica, elevação da } \\
\text { cabeceira a } 45^{\circ} \text { e pressão } \\
\text { cuff de } 25 \mathrm{~cm} \text { de } \mathrm{H}_{2} \mathrm{O} \text { ) } \\
\text { apresentaram } \\
\text { significância na } \\
\text { prevenção de PAV no } \\
\text { grupo de intervenção } \\
(p<0,05) \text {. }\end{array}$ & $\begin{array}{l}\text { O grupo intervenção } \\
\text { resultou em menor } \\
\text { índice de infecção } \\
\text { comparado ao } \\
\text { grupo controle que } \\
\text { não utilizou as } \\
\text { técnicas usuais. }\end{array}$ \\
\hline $\mathrm{VII}^{(12)}$ & $\begin{array}{l}\text { Leddye, } \\
\text { Wilkinson }\end{array}$ & 2015 & $\begin{array}{l}\text { Analisar a prática } \\
\text { de sucção e o uso } \\
\text { de salina na AET em } \\
\text { pacientes com VAA, } \\
\text { executada por } \\
\text { enfermeiros e } \\
\text { fisioterapeutas. }\end{array}$ & $\begin{array}{l}\text { Pesquisa avaliativa, } \\
\text { por meio de } \\
\text { questionário, sobre o } \\
\text { conhecimento e a } \\
\text { prática de aspiração e } \\
\text { o uso de salina, } \\
\text { aplicado a dois } \\
\text { grupos: enfermeiros e } \\
\text { fisioterapeutas. }\end{array}$ & $\begin{array}{l}\text { Ambos os grupos } \\
\text { notaram efeitos adversos } \\
\text { no paciente após } \\
\text { administração de salina } \\
\text { (desconforto, queda de } \\
\text { saturação e aumento da } \\
\text { frequência cardíaca). }\end{array}$ & $\begin{array}{l}\text { Não recomendado o } \\
\text { uso de salina na } \\
\text { AET, e sugerida } \\
\text { capacitação } \\
\text { profissional para } \\
\text { este procedimento. }\end{array}$ \\
\hline $\mathrm{VIII}^{(10)}$ & $\begin{array}{l}\text { Nesami- } \\
\text { Bagheri et } \\
\text { al. }\end{array}$ & 2015 & $\begin{array}{l}\text { Avaliar a qualidade } \\
\text { da implementação } \\
\text { de medidas } \\
\text { preventivas de PAV } \\
\text { nas UTI's de } \\
\text { hospitais } \\
\text { universitários. }\end{array}$ & $\begin{array}{l}\text { Estudo transversal, } \\
\text { realizado com } \\
\text { profissionais de } \\
\text { enfermagem que } \\
\text { atuam diretamente } \\
\text { com pacientes em } \\
\text { VM, em três UTI's } \\
\text { universitárias. }\end{array}$ & $\begin{array}{l}\text { Houve a necessidade de } \\
\text { capacitação para todos os } \\
\text { profissionais envolvidos, } \\
\text { quanto às medidas } \\
\text { preventivas de PAV. }\end{array}$ & $\begin{array}{l}\text { O conhecimento } \\
\text { adquirido em } \\
\text { medidas preventivas } \\
\text { gerou promoção da } \\
\text { qualidade e } \\
\text { segurança na prática } \\
\text { assistencial. }\end{array}$ \\
\hline$I X^{(17)}$ & $\begin{array}{l}\text { Dastdadeh } \\
\text { et al. }\end{array}$ & 2016 & $\begin{array}{l}\text { Comparar os efeitos } \\
\text { da AET por ASF e } \\
\text { ASA, considerando } \\
\text { a agitação e dor de } \\
\text { pacientes em VM } \\
\text { internados na UTI. }\end{array}$ & $\begin{array}{l}\text { Ensaio clínico } \\
\text { randomizado, com } \\
\text { pacientes, } \\
\text { distribuídos em dois } \\
\text { grupos: } \\
\text { ASF e ASA. }\end{array}$ & $\begin{array}{l}\text { Os pacientes } \\
\text { apresentaram aumento } \\
\text { na evolução de dor e } \\
\text { agitação em cada um dos } \\
\text { grupos ( } p<0,001) \text {, porém } \\
\text { não houve diferença } \\
\text { significante entre eles } \\
(p>0,05) \text {. }\end{array}$ & $\begin{array}{l}\text { Não foi constatado } \\
\text { diferença entre os } \\
\text { grupos quanto ao } \\
\text { nível da dor e } \\
\text { agitação. }\end{array}$ \\
\hline
\end{tabular}


A base de dados PubMed apresentou maior número de artigos. Por sua vez, o baixo número de produções na base de dados LILACS destaca a escassez de publicações brasileiras sobre o tema na enfermagem, quanto às técnicas de AET, conforme as frequências apresentadas na Tabela 1.

Tabela 1 - Frequência e percentual da distribuição dos artigos, segundo as bases de dados. Curitiba, Paraná, 2017.

\begin{tabular}{l|c|c}
\hline Base de dados & Quantidade (n) & Percentual (\%) \\
\hline LILACS & 2 & 13,3 \\
PubMed & 13 & 86,7 \\
Total & 15 & 100,0 \\
\hline
\end{tabular}

Fonte: Os autores (2017).

No que se refere aos periódicos dos estudos elencados, $100 \%$ são internacionais, tendo apresentado contribuição de publicações da enfermagem em AET, conforme o objetivo desta pesquisa. A Tabela 2 apresenta os periódicos selecionados e a respectiva quantidade de artigos usados nesta revisão.

Tabela 2 - Frequência e percentual de artigos, segundo o periódico selecionado. Curitiba, Paraná, 2017.

\begin{tabular}{|c|c|c|}
\hline Periódico & Quantidade (n) & Percentual (\%) \\
\hline Anesthesiology and Pain Medicine & 1 & 11,1 \\
\hline Canadian Journal of Respiratory Therapy & 1 & 11,1 \\
\hline Iranian Journal of Nursing and Midwifery Research & 4 & 44,5 \\
\hline Journal of Caring Sciences & 1 & 11,1 \\
\hline Journal of Clinical and Diagnostic Research & 1 & 11,1 \\
\hline Journal of Research in Pharmacy Practice & 1 & 11,1 \\
\hline Total & 9 & 100,0 \\
\hline
\end{tabular}

Fonte: Os autores (2017).

Após a análise minuciosa dos nove artigos apresentados na Figura 2, observou-se que as intervenções de enfermagem estão diretamente relacionadas à minimização de complicações referentes a alterações hemodinâmicas, respiratórias e dos gases sanguíneos, pressão de sucção, instilação de solução salina, hiperoxigenação, colonização microbiana, aspirações invasiva e minimamente invasiva, prevenção de infecção associada à VM, quantidade de secreção aspirada, experiência e conhecimento profissional na AET em pacientes com VAA em VM. Assim, foi possível evidenciar 16 intervenções de enfermagem na AET, destacadas e apresentadas por frequência de citação em cada estudo na Tabela 3.

Tabela 3 - Intervenções de enfermagem identificadas nos estudos selecionados sobre manejo na AET em pacientes com VAA em VM e frequência de citação. Curitiba, Paraná, 2017.

\begin{tabular}{|c|c|c|}
\hline \multirow{2}{*}{ Intervenção de enfermagem } & \multicolumn{2}{|c|}{ Frequência ( $n=16)$} \\
\hline & Absoluta (f) & Relativa (\%) \\
\hline Aspiração bucal & 1 & 6,3 \\
\hline AET por pressão de até $200 \mathrm{mmHg}$ por sistema fechado & 1 & 6,3 \\
\hline AET por pressão de até $200 \mathrm{mmHg}$ por sistema aberto & 1 & 6,3 \\
\hline Aspiração invasiva do tubo endotraqueal & 2 & 12,6 \\
\hline Aspiração minimamente invasiva do tubo endotraqueal & 2 & 12,6 \\
\hline Aspiração subglótica & 1 & 6,3 \\
\hline Hiperoxigenação antes e após a ASF & 1 & 6,3 \\
\hline Hiperoxigenação antes e após a ASA & 1 & 6,3 \\
\hline Instilação de salina na VAA & 1 & 6,3 \\
\hline Lavagem das mãos antes da aspiração da CET & 2 & 12,6 \\
\hline Manter cabeceira da cama hospitalar elevada em $30^{\circ}$ & 1 & 6,3 \\
\hline Manter cabeceira da cama hospitalar elevada em $45^{\circ}$ & 2 & 12,6 \\
\hline Manter pressão do cuff em $25 \mathrm{cmde} \mathrm{H}_{2} \mathrm{O}$ & 2 & 12,6 \\
\hline $\begin{array}{l}\text { Monitorar frequências cardíaca e respiratória, } \mathrm{SpO}_{2} \text { e pressão arterial do } \\
\text { paciente durante e após a aspiração invasiva do tubo orotraqueal }\end{array}$ & 1 & 6,3 \\
\hline
\end{tabular}


Realizar higiene oral com gluconato de clorexedine Realizar sucção na técnica estéril

Fonte: Os autores (2017).

Com a análise minuciosa dos artigos selecionados, considerando as intervenções e o manejo da enfermagem na AET em pacientes com VAA em VM invasiva, observou-se que, ao comparar as técnicas de aspiração baseadas em pesquisas e as usuais, os resultados foram mais eficazes nos grupos de intervenção $(p<0,05)$ do que nos de controle $(p>0,05)^{(11,14-16)}$, o que reforça a necessidade de a equipe de enfermagem utilizar técnicas na $A E T$, respaldadas por estudos experimentais ${ }^{(9,10,12)}$, para garantir a eficácia e a segurança do procedimento a ser executado no paciente crítico em UTI.

Quanto à aspiração subglótica no pacote de prevenção de $\operatorname{PAV}^{(11)}$, os resultados $(p<0,05)$ evidenciaram que a técnica apresentou menor ocorrência de risco de PAV no grupo de intervenção (redução de secreção entre o cuff do tubo orotraqueal e a mucosa traqueal, minimizando a colonização bacteriana), em comparação com o grupo de controle, que não utilizou o pacote de prevenção. Esse desfecho é compatível com o resultado da revisão de literatura ${ }^{(21)}$, o qual descreve que a aspiração subglótica proporciona benefícios aos pacientes críticos, uma vez que reduz a incidência de PAV de início precoce e consequentemente, os custos hospitalares.

Com relação à hiperoxigenação antes e após o procedimento de AET por sistemas aberto e fechado ${ }^{(13,14)}$, os estudos apresentaram que essa técnica contribui para a redução da chance de hipóxia no paciente durante a aspiração, minimizando complicações do procedimento. Um ensaio clínico randomizado ${ }^{(22)}$ evidenciou que a hiperoxigenação com elevação das Frações Inspiradas de Oxigênio $\left(\mathrm{FiO}_{2}\right) 50 \%$ acima da basal pode ser utilizada para prevenir a hipoxemia durante a AET em pacientes adultos e estáveis em VM, principalmente aqueles ventilados com valores de Pressão Positiva Expiratória Final (PEEP) reduzidos.

No tocante à aspiração por pressão de até $200 \mathrm{mmHgpor}$ sistemas aberto e fechado ${ }^{(13,14)}$, os autores dos estudos apontaram que essa escolha colaborou na minimização de acúmulo de secreções pulmonares e residuais na VAA. Esse valor de pressão não apresentou efeitos prejudiciais nas funções cardiorrespiratórias de indivíduos em VM, porém foi observado que as alterações hemodinâmicas recorrentes na aspiração retornaram aos seus índices basais, em menor tempo no grupo de ASF. Por sua vez, em análise comparativa randomizada entre dois tipos de sistema de aspiração traqueal (aberto e fechado) em recém-nascidos ${ }^{(23)}$, não foram evidenciadas diferenças estatísticas significantes entre os grupos. No entanto, esses autores afirmaram que, em pacientes com doenças pulmonares mais graves, como é o caso daqueles com hérnia diafragmática que cursa com hipertensão pulmonar persistente, o uso do sistema fechado pode ser mais benéfico.

Em relação aos cuidados da técnica de aspiração bucal $^{(11)}$, higiene oral com gluconato de clorexedine, elevação da cabeceira de $30^{\circ}$ a $45^{\circ}$, higiene das mãos antes da aspiração, controle da pressão do cuff em $25 \mathrm{~cm}$ de $\mathrm{H}_{2} \mathrm{O}$, aspiração na técnica estéril e frequência de sucção, os estudos $^{(9-11)}$ analisados ressaltaram serem imprescindíveis nas práticas assistenciais dos enfermeiros durante a $A E T$, para a minimização de riscos de PAV e de colonização bacteriana em vias aéreas de pacientes com VAA. Essas técnicas podem promover qualidade e segurança no cuidado ao paciente com assistência ventilatória invasiva em UTI ${ }^{(24)}$, sendo que grande parte dos cuidados mencionados possui evidências científicas quanto à sua utilização ${ }^{(21,24,25)}$.

Sobre a instilação de solução salina na VAA do paciente no momento da $\mathrm{AET}^{(12)}$, abordou-se o entendimento dos profissionais enfermeiros $\mathrm{e}$ fisioterapeutas sobre essa técnica, e foi constatado desconhecimento do risco, benefício e indicação, fato que gerou a avaliação quanto à necessidade da capacitação desses profissionais e a necessidade da implantação da avaliação de rotina antes do procedimento, visto que esta não estava vinculada a uma prática diária. Outros estudos apontaram ${ }^{(8,26)}$ que o deficit de conhecimento dessa técnica pelos profissionais da saúde pode causar complicações ao paciente grave na $A E T$, tais como: hipoxemia, instabilidade hemodinâmica e infecções, além do desconforto respiratório do indivíduo; assim, a instilação de solução salina não é indicada rotineiramente.

Ainda, o desfecho de uma revisão sistemática ${ }^{(27)}$ apresentou que a instilação de solução salina em volume de $5 \mathrm{ml}$ é eficaz na diminuição de ocorrência de PAV, comparada à 
não instilação. Há, portanto, necessidade de avaliação clínica e exame físico criterioso pelo enfermeiro na sua indicação na AET. Essa avaliação poderá ocorrer por meio do processo de enfermagem que, através dos diagnósticos do enfermeiro, poderá evidenciar os riscos e a prevenção de complicações secundárias às prática de enfermagem ${ }^{(28)}$.

Por fim, ao analisar os efeitos das aspirações minimamente invasivas e invasiva na AET, os estudos selecionados ${ }^{(15,16)}$ apresentaram significância entre os grupos $(p<0,05)$, prevalecendo maior eficácia naquele que utilizou a aspiração invasiva nos critérios de: minimização de secreção traqueal, maior permeabilidade da VAA, menor manipulação da traqueia $e$ periodicidade de aspiração. Os autores relataram que a periodicidade de aspiração invasiva foi menor, obtendo menor manipulação da traqueia e, consequentemente, menor risco de PAV. Entretanto, o aumento dos parâmetros de frequência cardíaca e pressão arterial nesses pacientes foi superior, comparado ao grupo de aspiração minimamente invasiva. Uma vez que esse aumento, mesmo em pequeno grau, pode causar eventos adversos em pacientes cardiopatas, os enfermeiros devem monitorar os parâmetros cardiovasculares durante e após a AET invasiva. Os autores também recomendaram estudos adicionais para comparar os efeitos fisiológicos nas AETs invasiva e minimamente invasiva.

Verificou-se que, a partir das intervenções encontradas por meio da análise dos estudos selecionados e das considerações apresentadas, comparadas a outros estudos na literatura, podese afirmar que existem evidências importantes e atuais sobre a técnica e o manejo da enfermagem na AET. Os artigos elegidos apresentam a eficácia das técnicas executadas pelos enfermeiros nos grupos experimentais e o risco de ocorrer insucesso na aspiração e complicações ao paciente, caso não seja executada por meio de técnicas baseadas em evidências científicas.

Para tanto, é necessária a elaboração, pela equipe de enfermagem, de protocolos assistenciais e capacitação dos profissionais em todo o processo da AET, por meio de uma literatura atualizada. Os artigos incluídos nesta revisão afirmam que a educação continuada deve cumprir uma vigilância contínua e eficaz entre as equipes de enfermagem, no intuito de contribuir na ampliação do conhecimento e, esse, efetivado na prática clínica de todos os envolvidos.
Frente ao exposto, evidencia-se a importância de fomentar pesquisas científicas sobre essa temática, com o objetivo de inovar as técnicas destacadas na literatura pesquisada e necessárias na aplicação diária na prática dos profissionais diretamente envolvidos nesse procedimento.

\section{CONCLUSÃO}

Por meio desta pesquisa, foi possível apresentar uma revisão integrativa das intervenções de enfermagem nos cuidados e manejo na AET em pacientes com VAA em VM. Nesse contexto, faz-se necessária a avaliação do paciente pelo enfermeiro antes, durante e após o procedimento, por meio do exame físico e monitoração, para que possa selecionar a melhor técnica, possibilitando tomar decisões de cuidado na AET com segurança. Ainda, são imprescindíveis a atualização técnico-científica e a participação dos profissionais enfermeiros no planejamento do cuidado ao paciente crítico, sendo as técnicas apresentadas compatíveis com outros estudos experimentais e quantitativos disponíveis na literatura.

Durante o processo de análise dos artigos, foi observada a limitação de estudos experimentais da enfermagem, tratando da inovação de cuidados na $A E T$, principalmente de referências nacionais sobre o tema. Cabe ressaltar a importância do desenvolvimento de pesquisas de intervenções de enfermagem na $A E T$ e no cuidado ao paciente crítico com VAA em VM, que possibilitem a escolha da técnica ideal para esse procedimento, com base em evidência científica, tornando o cuidado seguro. Com isso, não se expõe o paciente ao risco, visando à qualidade da sua assistência e minimizando complicações secundárias na AET.

Esta revisão deve ser de grande incentivo para pesquisas futuras sobre a elaboração de estudos experimentais envolvendo as intervenções de enfermagem na $A E T$, visto que os resultados dos cuidados analisados e discutidos reforçam a qualidade e a segurança nesse procedimento.

\section{REFERENCES}

1. Martins R, Nunes PM, Xavier PA, Wittkopf PG, Schivinski CIS. Aspiração traqueal: a técnica e suas indicações. ACM Arq Catarinense Med. 2014 [citado em 05 jan 2017];43(1):90-6. Disponível em: 
http://www.acm.org.br/revista/pdf/artigos 1280.pdf

2. Amatéa SL, Piva JP, Zanella MI, Bruno F, Garcia PCR. Acesso rápido à via aérea. J Pediatr. (Rio J). 2003;79(Supl. 2):S127S138. $\quad$ https://doi.org/10.1590/S002175572003000800002

3. Yousefnia-Darzi F, Hasavari F, Khaleghdoost T, Kazemnezhad-Leyli E, Khalili M Effects of thoracic squeezing on airway secretion removal in mechanically ventilated patients. Iran J Nurs Midwifery Res. 2016 May-Jun;21(3):337-42.

https:// doi.org/10.4103/1735-9066.180374

4. American Association for Respiratory Care. AARC clinical practice guidelines: endotracheal suctioning of mechanically ventilated patients with artificial airways 2010. Respir Care. 2010 Jun;55(6):758-64.

5. Martins JJ, Maestri E, Dogenski D, Nascimento ERP, Silva RM, Gama FO. Necessidade de aspiração de secreção endotraqueal: critérios utilizados por uma equipe de enfermagem de uma unidade de terapia intensiva. Cienc Cuidado Saunecesside. 2008;7(4):517-22. doi: https://doi.org/10.4025/cienccuidsaude.v7i $\underline{4.6660}$

6. Conselho Federal de Enfermagem (COFEN). Resolução № 0557/2017. Normatiza a atuação da equipe de enfermagem no procedimento de Aspiração de Vias Aéreas. Brasília, DF: Conselho Federal de Enfermagem. 2017 [citado em 12 dez 2017]. Disponível em: www.cofen.gov.br/resolucao-cofen-no05572017 54939.html

7. Ferreira AOM, Lima DVM, Christovam BP, Silvino ZR. Aspiração endotraqueal em unidade de terapia intensiva: uma revisão integrativa. Rev Enferm UFPE on Line. 2013 jul;7(7):4910-7.

https://doi.org/10.5205/1981-8963v7i7a11750p4910-4917-2013

8. Frota OP, Loureiro MDR, Ferreira AM. Aspiração endotraqueal por sistema aberto: práticas de profissionais de enfermagem em terapia intensiva. Esc Anna Nery. 2014;18(2):296-302.

\section{https://doi.org/10.5935/1414-} 8145.20140043

9. Hamishehkar $H$, Vahidinezhad $M$, Mashayekhi SO, Asgharian P, Hassankhani $\mathrm{H}$, Mahmoodpoor A. Education alone is not enough in ventilator associated pneumonia care bundle compliance. J Res Pharm Pract. 2014 Apr;3(2):51-5. https://dx.doi.org/10.4103/2279042X.137070

10. Bagheri-Nesami M1, Amiri-Abchuyeh M2, Gholipour-Baradari A3, YazdaniCherati J4, Nikkhah A. Assessment of critical care provider's application of preventive measures for ventilatorassociated pneumonia in intensive care units. J Clin Diagn Res. 2015 Aug;9(8):IC05IC08.

https://doi.org/10.7860/JCDR/2015/11996. $\underline{6308}$

11. Bakhtiari S, Yazdannik A, Abbasi S, Bahrami N. The effect of an upper respiratory care program on incidence of ventilator-associated pneumonia in mechanically ventilated patients hospitalized in intensive care units. Iran J Nurs Midwifery Res. 2015 May-Jun [citado em 05 fev 2017];20(3):354-8. Disponível em:

https://www.ncbi.nlm.nih.gov/pubmed/261 20336

12. Leddy R, Wilkinson JM. Endotracheal suctioning practices of nurses and respiratory therapists: how well do they align with clinical practice guidelines? Can J Respir Ther. 2015 Summer [citado em 10 fev 2017];51(3):60-4. Disponível em: https://www.ncbi.nlm.nih.gov/pmc/article s/PMC4530836/pdf/cjrt-51-60.pdf

13. Yazdannik AR, Haghighat $S$, Saghaei $M$, Eghbali M. Comparing two levels of closed system suction pressure in ICU patients: evaluating the relative safety of higher values of suction pressure. ran J Nurs Midwifery Res. 2013 Mar [citado em 20 jan 2017]; 18(2):117-22. Disponível em: https://www.ncbi.nlm.nih.gov/pubmed/239 $\underline{83740}$ 
14. Yousef H, Vahdatnejad J, Yazdannik AR. Comparison of the effects of two levels of negative pressure in open endotracheal tube suction on the physiological indices among patients in intensive care units. Iran J Nurs Midwifery Res. 2014 Sep [citado em 25 jan 2017];19(5):473-. Disponível em: https://www.ncbi.nlm.nih.gov/pubmed/254 $\underline{00674}$

15. Abbasinia $M$, Irajpour A, Babaii $T$, Shamali M, Vahdatnezhad J. Comparison the effects of shallow and deep endotracheal tube suctioning on respiratory rate, arterial blood oxygen saturation and number of suctioning in patients hospitalized in the intensive care unit: a randomized controlled trial. J Caring Sci. $2014 \quad$ Sep 1;3(3):157-64. https://doi.org/10.5681/jcs.2014.017

16. Irajpour $A$, Abbasinia $M$, Hoseini $A$, Kashefi P. Effects of shallow and deep endotracheal tube suctioning on cardiovascular indices in patients in intensive care units. Iran J Nurs Midwifery Res. 2014 Jul [citado em 15 fev 2017];19(4):366-70. Disponível em: https://www.ncbi.nlm.nih.gov/pubmed/251 83976

17. Dastdadeh R, Ebadi A, Vahedian-Azimi A. Comparison of the effect of open and closed endotracheal suctioning methods on pain and agitation in medical ICU patients: a clinical trial. Anesth Pain Med. 2016 Jul 31;6(5):e38337. eCollection 2016 Oct. https://doi.org/10.5812/aapm.38337

18. Souza MT, Silva MD, Carvalho R. Revisão integrativa: o que é e como fazer. Einstein (Sao Paulo). 2010;8(1):102-6. https://doi.org/10.1590/s1679-

45082010rw1134

19. Mendes KDS, Silveira RCCP, Galvão CM. Revisão integrativa: método de pesquisa para a incorporação de evidências na saúde e na enfermagem. Texto Context Enferm. 2008;17(4):758-64. doi: $\quad$ https://doi.org/10.1590/S010407072008000400018

20. Moher D, Liberati A, Tetzlaff J, Altman DG, The PRISMA Group. Preferred reporting items for systematic reviews and meta-analyses: the PRISMA statement. PLoS Med. 2009 Jul 21;6(7):e1000097. https://doi.org/10.1371/journal.pmed.100 0097

21. Souza CR, Santana VTS. Impacto da aspiração supra-cuff na prevenção da pneumonia associada à ventilação mecânica. Rev Bras Ter Intensiva. 2012;24(4):401-6.

https://doi.org/10.1590/S0103507X2012000400018

22. Diniz GCLM, Souza AO, Oliveira HMD, Arrais RCS, Pessoa BP, Figueiredo PHS. A utilização de $\mathrm{FiO}_{2}$ inferior a $100 \%$ para hiperoxigenação de pacientes estáveis submetidos à aspiração endotraqueal. ASSOBRAFIR Cienc. 2012 [citado em 30 jan 2017];3(1):45-56. Disponível em: http://www.uel.br/revistas/uel/index.php/ rebrafis/article/view/10886/10598

23. Paula LCS, Ceccon MEJ. Análise comparativa randomizada entre dois tipos de sistema de aspiração traqueal em recémnascidos. Rev Assoc Med Bras. 2010;56(5):434-9. https://doi.org/10.1590/S0104$\underline{42302010000400016}$

24. Silva SG, Nascimento ERP, Salles RK. Bundle de prevenção da pneumonia associada à ventilação mecânica: uma construção coletiva. Texto Context Enferm. 2012;21(4):837-44.

https://doi.org/10.1590/S010407072012000400014

25. Frota OP, Loureiro MDR, Ferreira AM. Conhecimento sobre aspiração endotraqueal de profissionais de enfermagem: estudo descritivo. Online Braz J Nurs. 2013 [citado em 15 fev 2017];12(3):1-8. Disponível em: http://www.objnursing.uff.br/index.php/n ursing/article/viewFile/4115/2250

26. Xavier CC, Carmo AFS, Korinfsky JP, Nunes GFO, Silva RM, Mendes RNC. Enfermagem na assistência ventilatória: análise da aspiração endotraqueal na unidade de terapia intensiva. Rev Enferm UFPE on Line. 2013;7(12):6800-7. 
https://doi.org/10.5205/1981-8963-

v7i12a12341p6800-6807-2013

27. Favretto DO, Silveira RCCP, Canini SRMS, Garbin LM, Martins FTM, Dalri MCB. Aspiração endotraqueal em pacientes adultos com via aérea artificial: revisão sistemática. Rev Latino-Am Enferm. 2012;20(5):997-1007. https://doi.org/10.1590/S010411692012000500023

28. Ramos GS, Santana LC, Ferreira PHC, Chianca TCM, Guedes HM. Diagnósticos de enfermagem documentados em prontuários de pacientes em unidades de terapia intensiva. Rev Enferm Cent Oeste Mineiro. 2013;3(2):679-86. https://doi.org/10.19175/recom.v0i0.382

Nota: Trabalho resultante da Disciplina de Enfermagem em Unidade de Terapia Intensiva, da Graduação de Enfermagem do Centro Universitário Unidombosco, Curitiba-Paraná. Esse estudo não contou com apoio financeiro de agências de fomento à pesquisa.

Recebido em: 07/05/2017

Aprovado em: 13/03/2018

Endereço de correspondência:

Vagner José Lopes

Rodovia Br. 116 no 17844 Bloco 3 Ap 404 - Pinheirinho

CEP: 81690-410 - Curitiba/PR - Brasil

E-mail: profvagnerjlopes@gmail.com 